\title{
Task Decision Support System Research and design of Space Maritime TT\&C
}

\author{
Duan Huifen ${ }^{1, a^{*}}$, Bao Junlei ${ }^{1, a}$ and Zhang Dehua ${ }^{1, a}$ \\ 1.Satellite Marine Tracking and Control Department of China, Jiangyin , 214431, China
}

as5public@163.com

\section{Keywords: Decision Support; Data Mining; Space TT\&C}

Abstract. In order to improve the efficiency of the command of the maritime aerospace measurement and control tasks, the timely discovery and analyzing and solving the abnormal situation in the process of the task, research and design the assistant decision system which be appropriate for the task of the maritime measure control. Through mining and intelligent analysis the information, combining the characteristics of maritime aerospace measurement and control tasks, solved the problem that analyzing and judging the information reliance on artificial in the past, given the structure and function design which be to establish the task decision support system for space maritime TT\&C.

\section{Introduction}

Aerospace survey ships is an important part of TT\&C[1] network in our country, which has a strong comprehensive monitoring and control capabilities, characteristics of array flexible, can provide service of maritime TT\&C for various space missions. In the aerospace measurement and control task, large amounts of information of sea voyage, the marine hydrometeorological, the equipment fault, the test data of the target aircraft and the Results of data analysis. In order to minning the application value of the test information better, accumulate experience for the following task, it is necessary to carry out the research work for the maritime TT\&C decision support system (TDSS).

\section{System Analysis}

Main purpose of TDSS is to provide the global data analysis, and the situation display support in the process of the maritime TT\&C tasks, provide a basis for commander to timely and accurately for scientific decision-making. TDSS runs through the whole process of the test task, confirm and verify the scheme of the maritime TT\&C before the task, analyse and monitor the target in the task, analyse and dispose and evaluate the results after the task.

Measurement and Control Scheme Deduction. The position of the survey ship is accorded to the requirement of the task, which is different from the fixed and precise location of the ground-based TT\&C[2]. Designing the measuring conditions of the survey ship before the task, avoiding the shade between hull and tracking antenna, the ocean currents and wind effects on course, the influence of the ship hull resonance to the safety of the ship and the equipment, the influence and effect of comprehensive calibration of inertial navigation system on the positioning precision of the site, the influence of the track geometry design's on the accuracy of measurement. The weather of the task waters changing hugely, when the conditions do not meet the requirement of the TT\&C, it needs to weigh the avoiding the risk of sailing between the completing the task, choose the best scheme, simulate the scheme, adjust the plan and working process synchronously. The specific features include:

1) measuring condition of deduction: considering synthetically the requirements of the navigation safety, the task segment, the direction pattern coverage of the antenna, the work perspective of the tt\&c communication, the measurement and control accuracy of the data,designing the best position, heading, speed and route, calculating the tracking performance parameters of the equipment to the target. Through simulation calculation, process demonstration, and comparison of several sets of schemes, the feasibility and optimality of the scheme design is verified. 
2) generate the plan of the measurement and control: according to the measurement and control plan of the center, generating the latest measure and control plan dynamically in real time.

3) generate the task flow: according to the measure and control plan and the specification of the survey ship in the task, generating the ship's work flow in real time, and guiding the trial work.

Surveillance, Judgment and Analysis of The Target.The core task of the maritime TT\&C is to complete the measurement and control communication tasks[3] of the target, according to the requirements of spacecraft and rocket trajectory and control, using the survey ship at the specified location in the sea. Using the known measurement information, according to the flight characteristics of the target to determine whether the target according to a predetermined orbit quickly, the key components of the target is working normally, to take timely measures to deal with. The main functions are as follows:

1) situation analysis: gets the information of the target position in real-time, and carries on the high accuracy smoothing, calculates the error between the theoretical trajectory and the real-time trajectory. Provides the basis for the judgment and the analysis of the flight situation.

2) aircraft health status analysis: through calculating the telemetry data of the aircraft, and comparing the calculation results of the key parameters in racking arc with the theoretical values, judge the key control events completed situation and the continuous change of the telemetry parameters in the tracking arc, so master the aircraft's health.

3) selecting the best initial orbit: calculates multiple sets of initial orbits according to the real-time measurement data, according to the selection rules for comparison, calculates the polymerization conditions of the initial orbits. Gives the comparison of all types of initial orbits with the theoretical orbit, and selects the best orbit.

Measurement and Control Process Analysis.In order to ensure the accuracy of the measurement and control equipment to capture and track the target, to ensure the accurate and reliable data transmission between space and ground, it is very important to analyze and judge the process of the measurement and control. By comparison, analysis and judgment of the measurement and control data, capture the anomaly of the monitoring and controlling process, and analyze the cause of the abnormal phenomenon. The main functions as follows:

1) optimization of the equipment tracking and guidance information: according to the comparison results between the theory data with the measured data, then select the guide information of the tracking equipment.

2) space ground link analysis: the survey ship in tracking, according to the target antenna pattern and compute the change of the target antenna gain in tracking arc. according to the calculation results, analyze the change of the signal to noise ratio belongs to the up-downlink, judge the correct link whether it can guarantee the right information transmission between space and ground for the measurement and control.

3 ) the quality of measurement data analysis: Define the data anomaly judgment rules, Capture and record abnormal data information in real time. Collect the condition of the data reception, tracking, abnormal, provide the analysis tools for the abnormal data, analyze the cause of the abnormal data.

4) the effect of equipment failure analysis: call the remote test fault diagnosis system, using the fault tree of the system, analyze the causes and effects range of the fault, find the same or similar equipment fault disposal methods ever.

Task Completion Status Assessment.After the completion of the task, it is need to collect and analyze the task requirements to meet, real-time monitoring, the analysis results, and with the same types of task analysis results for longitudinal comparison. Through the establishment of evaluation system, the evaluation of the task is carried out, which provides a reference for the following tasks. Specific features include:

1) Post rapid assessment: rapid analysis on disk data afterwards, Collect measurement accuracy, analyze guide data accuracy and precision of orbit determination, abnormal situation. Compared with the historical treatment results of the same type task, given the consistency of the analysis results with the precision. 
2) overall evaluation of the task: set up the evaluation index and algorithm. According to the processes of tasks, post training situation, quality monitoring results, data analysis results, quantify the task completion of effectiveness evaluation.

3) archiving the test information: to achieve the test information, and unify the information into the database, and provide the data for the complex play and analysis. Users can easily on the current task and historical task data for query, browse, export and archiving and other related operations, and provide text, charts and curves of various forms of information presentation for the decision analysis.

\section{System Design}

TDSS is a test task application system based on computer processing and analysis. The key is to establish the model of description and analysis to the problem. TDSS improves its ability to analyze and process the information through the preset model. All of its design, analysis and operation must be based on the preset model. It can not only effectively using original data, models, and methods to make a decision, but also reasoning and judgment in the decision making process, generate new data, models and methods for decision support services [4].

System Structure. TDSS using a number of different modules for data collection, comprehensive treatment, the logic of data processing is very complex, it is suitable to use the server to process the data. Commanders use the browser to Interact with the system, access to the decision. So the system uses the browser / server software architecture. The system structure of TDSS is shown in Figure 1.

The system has user layer, processing layer and data layer. The user layer uses the browser as the client, access the resources of the analyzing server through the orientation address. Processing layer and data layer deployed on the server, according to the user's access request, through the user access control, generate the analysis results by each subsystem , and then presented to the user.

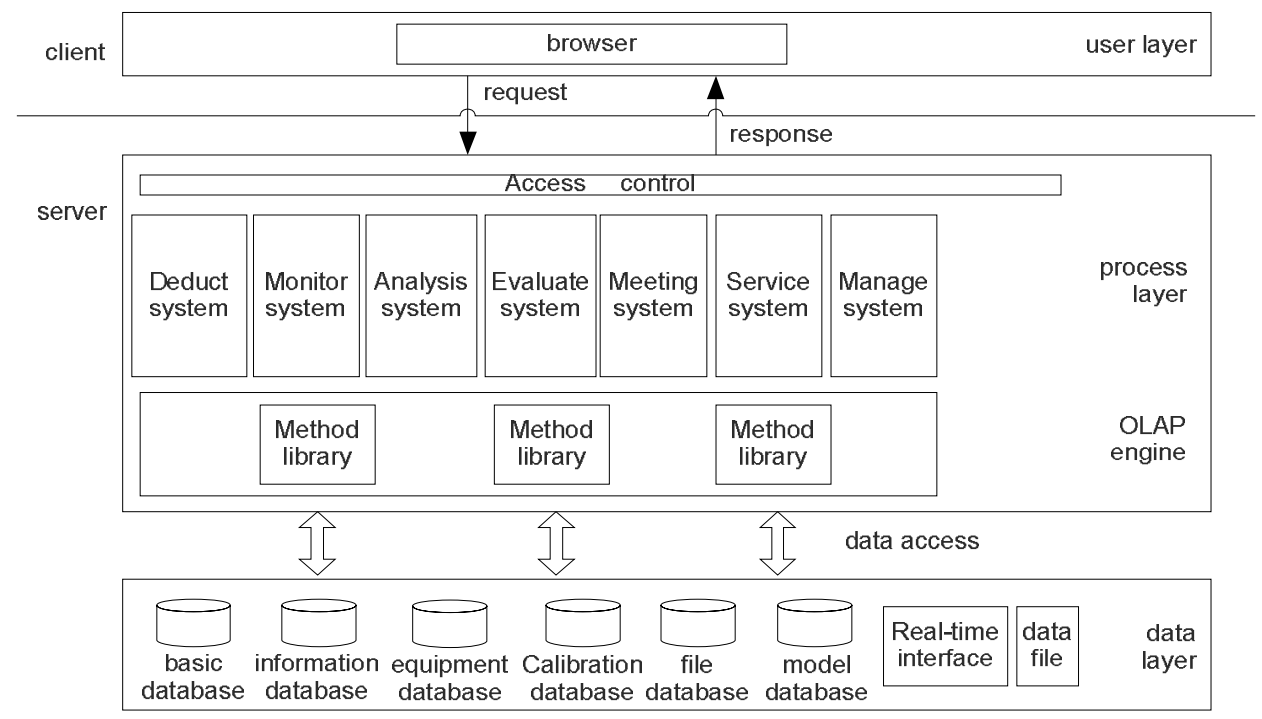

Figure 1 TDSS system structure

Assistant Analysis Process.The TDSS's analysis object is all kinds of test data, the data has standardized structure, stored in the database, the data file and the receiving real-time buffer. The TDSS assembles all standard calculation method to a method base, to dispose the original data of the analysis process. The analysis of the maritime TT\&C is based on the work of knowledge, especially the large amount of tacit knowledge, which plays an important role in the analysis of the results of the mission [5].The special knowledge which solving the semi-structured and non structured problem be assembled to be the knowledge base. Through logical combination of elements in the knowledge base, the library of knowledge reasoning model will be build, finally engender the scheme of qualitative and quantitative analysis method, to decision support. The model base includes not only the basic models that support different levels of computing and decision making, but also can form a new model based on the combination of modules. 
The TDSS's subsystem faces different problems, based on different knowledge rules, adopts the different model. So, it is necessary to construct each knowledge base and model base for specific problems, but the following decision-making process is similar. It can be summed up as the analysis process in Figure 2. Through the analysis of massive data mining, combined with the processing of artificial intelligence and the corresponding user intervention, thus forming the final analysis results.

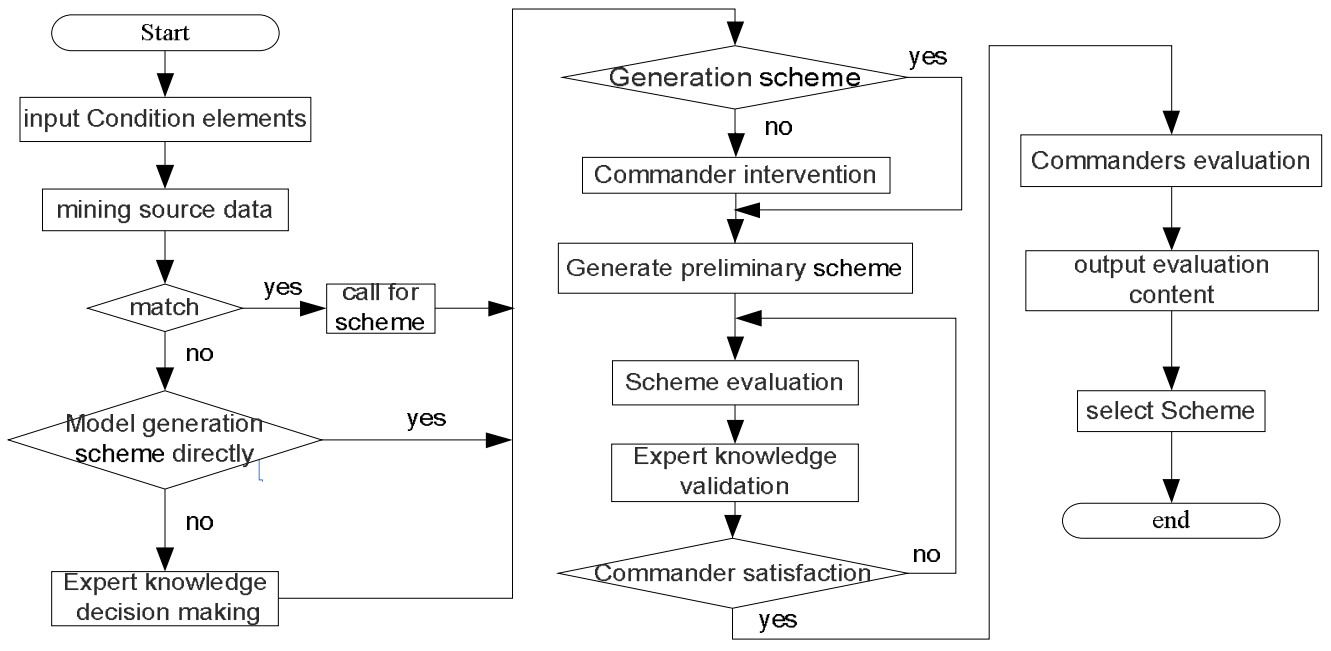

Figure 2 analysis process

\section{Summary}

The task decision support system for space maritime TT\&C includes 7 subsystems, such as scheme deduction, target monitoring and analysis, process analysis, task evaluation, information service and system management. According to the task requirement, the target characteristics and the survey ship tracking characteristics, by using the real-time data, historical data, and using the calculating method with difference, tatistical analysis, clustering processing, tracking of data association and rule ratiocination, the task decision support system makes quantitative analysis on the task, enhances the afterwards analysis to the real-time analysis, enhances the individual experience to the intelligence analysis. So as to improve the effectiveness of discovering, analyzing and solving problem, to serve the task of maritime TT\&C.

\section{References}

[1] Jiang Wenda et al:aerospace survey ships [M], Beijing defence press, 1996

[2] Jian Shilong, Fei Jiabing,Liu Bing: Introduction to technology of maritime TT\&C for aerospace survey ships [M], Beijing defence press, 2009.

[3] Zhao Wenhua: The technical terms of maritime TT\&C [M], Beijing defence press, 2013.

[4] Cao Lei, Bao Guangyu, Chen Guoyou: command information system [M],Beijing defence press, 2013.8 reprint .

[5] Huang Kai, Zhang Zhonghua, et al. Based on immune computing, multi Agent decision support system and its application in the field of maritime TT\&C[J] telecommunication technology, 2007 (04) forty-seventh volume second. 